\title{
Necrotizing pneumonia and sepsis due to Clostridium perfringens:
} a case report

\author{
Cristina Palmacci1 ${ }^{1}$, Manuela Antocicco ${ }^{1}$, Lorenzo Bonomo², Fabio Maggi², \\ Alberto Cocchi ${ }^{1}$ and Graziano Onder*1
}

\author{
Address: ${ }^{1}$ Department of Geriatrics, Policlinico A. Gemelli, Università Cattolica del Sacro Cuore, Rome, Italy and ${ }^{2}$ Department of Bioimaging and \\ Radiological Sciences, Policlinico A. Gemelli, Università Cattolica del Sacro Cuore, Rome, Italy \\ Email: Cristina Palmacci - palmacci_cristina@libero.it; Manuela Antocicco - manuela antocicco@rm.unicatt.it; \\ Lorenzo Bonomo - lbonomo@rm.unicatt.it; Fabio Maggi - fabio.maggi@rm.unicatt.it; Alberto Cocchi - alberto_cocchi@rm.unicatt.it; \\ Graziano Onder* - graziano_onder@rm.unicatt.it \\ * Corresponding author
}

Published: 14 January 2009

Cases Journal 2009, 2:50 doi:10.1186/1757-1626-2-50

This article is available from: http://www.casesjournal.com/content/2/I/50

(c) 2009 Palmacci et al; licensee BioMed Central Ltd.

This is an Open Access article distributed under the terms of the Creative Commons Attribution License (http://creativecommons.org/licenses/by/2.0), which permits unrestricted use, distribution, and reproduction in any medium, provided the original work is properly cited.

Received: 4 December 2008

Accepted: 14 January 2009

\begin{abstract}
Clostridia are uncommon causes of pleuropulmonary infection. Clostridial species infecting the pleuropulmonary structures characteristically cause a necrotizing pneumonia with involvement of the pleura. Most cases have iatrogenic causes usually due to invasive procedures into the pleural cavity, such as thoracentesis or thoracotomy, or penetrating chest injuries. Rarely clostridia pleuropulmonary infections are not related to these factors. The clinical course of pleuropulmonary clostridial infections can be very variable, but they may be rapid and fatal. We report a rare case of necrotizing pneumonia and sepsis due to Clostridium perfringens not related to iatrogenic causes or injuries in an 82 years old woman.
\end{abstract}

\section{Background}

Clostridia are uncommon causes of pleuropulmonary infection, especially in nonsurgical or nontraumatic patients [1]. Clostridial species infecting the pleuropulmonary structures characteristically cause a necrotizing pneumonia with involvement of the pleura. Most cases have iatrogenic causes: the Clostridia colonizing the skin by contamination may be introduced into the pleural space following invasive procedures, such as thoracentesis or thoracotomy, or penetrating chest injuries $[1,2]$. Other predisposing factors for necrotizing pneumonia are aspiration of oropharyngeal or gastric contents [1-4], pulmonary embolism with infarction (haematogenous seeding of infarcted lung tissue) [1]. In addition, this condition is often associated with chronic disease, such as diabetes or cirrhosis, and underlying pleuropulmonary pathology (pulmonary tuberculosis, chronic pleural effusions) [2,4]. Reported predisposing factors for systemic clostridial dissemination include intraoral pathology (carious teeth or gingival disease) and intrabdominal pathology such as malignancy and enteric vascular malformation or subdiaphragmatic infections $[5,6]$. The clinical course of pleuropulmonary clostridial infections can be very variable: clostridial pleuropulmonary infections are often indolent but they can be rapid and fatal [1].

\section{Clinical case}

We present the case of a 82-years-old caucasian female, affected by paroxysmal atrial fibrillation (dual chamber pace-maker implanted in 2004), hypertension, normochromic normocytic anemia, arthritis, osteoporosis, cholelithiasis, admitted to our department for worsening 
of general conditions, hyperpyrexia and respiratory failure. At physical examination the patient was non-cooperative, drowsy and showed poorly productive cough and hyperpyrexia. Blood pressure was $100 / 70 \mathrm{mmHg}$, with a regular pulse rate of $90 \mathrm{bmp}$. Blood gas analysis showed hypoxemia (paO2: $50.5 \mathrm{mmHg}$ ) and hypocapnia (pCO2: $33.1 \mathrm{mmHg}$ ). Chest examination showed abolition of vesicular murmur over the lower part of the left hemi-thorax and of the right lower lung fields. Abdominal examination showed presence of a voluminous right inguinal hernia. Laboratory examination documented neutrophilic leukocytosis and renal failure.

The chest X-ray showed aspecific findings of bilateral pleural effusion with consolidation of lower lobes and lingula. It was therefore undertaken oxygen therapy, hydrating, diuretic and empirical antibiotic therapy with cephalosporins and macrolides and blood cultures were performed. After 48 hours, a multislice CT scan of the chest was performed, which confirmed bilateral pleural effusion and consolidation of lower lobes and lingula; in right lower lobe and lingula areas of cavitation were also demonstrated, suggesting the hypothesis of a necrotizing, gangrenous pneumonia (Figure 1). An abdominal ultrasound did not show any relevant finding.

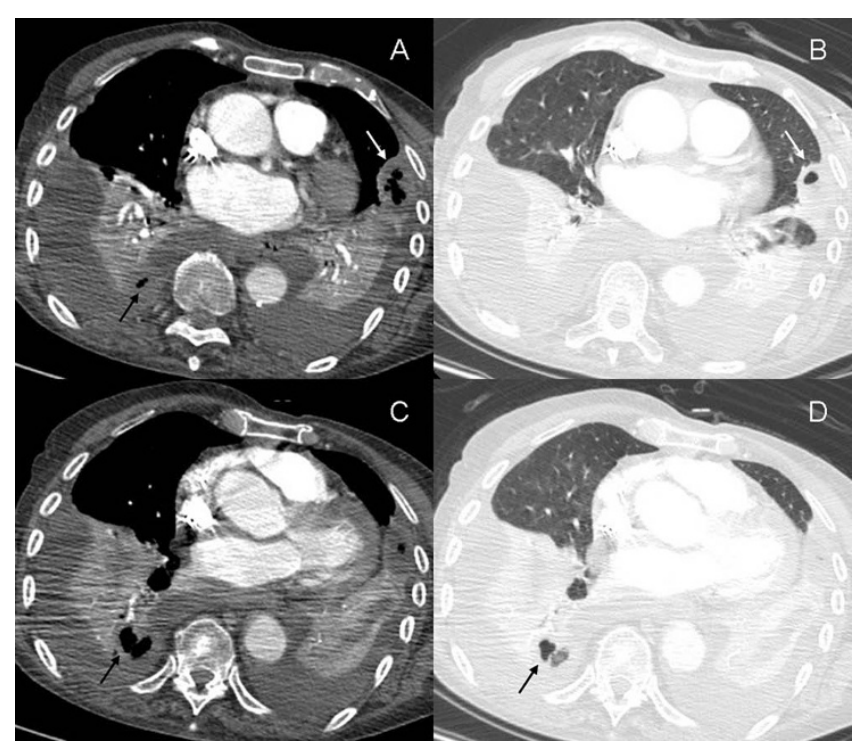

Figure I

Contrast-enhanced multi-slice CT of the thorax performed 48 hours after admission. Multi-slice CT of the thorax confirmed presence of bilateral pleural effusion and consolidation of lower lobes and lingula; cavitation was revealed in the pleural-based consolidation of the lingula (Figure $2 A-B$, white arrows) and in the right lower lobe (Figure 2A-C-D, black arrows). These findings were considered indicative of a necrotizing, gangrenous pneumonia.
Clostridium perfringens was identified from two blood cultures and combined antibiotic treatment with Clindamicin and Amoxiciliin/Clavulanate was started. In the following weeks patient health conditions improved and a CT scan of the chest performed after two weeks of treatment showed marked reduction of pleural effusion and of extent of parenchymal consolidation with disappearance of cavitating areas. Antibiotic treatment was continued for two months. After this period, the patient fully recovered and she did not show any sign or symptom of pleuropulmonary disease.

\section{Discussion}

Spontaneous pneumonia related to clostridium perfringens has rarely been described in the medical literature, while this condition seems more commonly associated with invasive procedures or penetrating chest injuries. Population based laboratory surveillance for invasive clostridium perfringens disease conducted in the population of the Calgary Health Region show an annual incidence of 0.83 per 100,000 with a striking age-related increased risk for acquisition [3]. This study shows that patients aged 65 years or older are at 12-fold higher risk for acquiring these infections as compared to younger patients [3].

Other risk factors for clostridium perfringens pneumonia are aspiration of oropharyngeal or gastric contents, pulmonary embolism with infarction (hematogenous seeding of infarcted lung tissue). In addition, this condition is often associated with chronic disease, such as diabetes or cirrhosis, and underlying pleuropulmonary pathology (pulmonary tuberculosis, chronic pleural effusions) is often found. Reported predisposing factors for systemic clostridial dissemination also include malignancies, in particular colon cancer. In this case, a endoscopy was not performed because the patient and her relatives refused the procedure.

\section{Consent}

Written informed consent was obtained from the patient relative for publication of this case report and accompanying images. A copy of the written consent is available for review by the Editor-in-Chief of this journal.

\section{Competing interests}

The authors declare that they have no competing interests.

\section{Authors' contributions}

$\mathrm{CP}, \mathrm{MA}, \mathrm{AC}, \mathrm{FL}$ and $\mathrm{GO}$ analyzed and interpreted the patient data regarding the infective disease. LB and FM performed and interpreted the chest X-ray and the CT scan. $\mathrm{CP}, \mathrm{MA}$ and GO were responsible for writing the manuscript. All authors read and approved the final manuscript. 


\section{References}

I. Spagnuolo PJ, Payne VD: Clostridial pleuropulmonary infection. Chest 1980, 78:622-5.

2. Boersma WG, Jag EJ van der, Holloway Y, Postmus P: Pleural empyema caused by Clostridium perfringens. Resp Med 1994, 88:787-8.

3. Jackson S, Gregson DB, McFadden S, Laupland KB: Clostridium perfringens pleuropulmonary infection and septic shock: case report and population-based laboratory surveillance study. Scand J Infect Dis 2003, 35:883-6.

4. Hardison JE: Primary clostridial pneumoni and empyema. Chest 1970, 57:390-2.

5. Tanabe KK, Jones WG, Barie PS: Clostridial sepsis and malignant disease. Surg Gynecol Obstet 1989, 169:423-8.

6. Craven CM: Fatal Clostridium perfringens septicemia associated with gastrointestinal arteriovenous malformations (vascular ectasias). Arch Pathol Lab Med 1989, I I3:534-5.

Publish with Bio Med Central and every scientist can read your work free of charge

"BioMed Central will be the most significant development for disseminating the results of biomedical research in our lifetime. "

Sir Paul Nurse, Cancer Research UK

Your research papers will be:

- available free of charge to the entire biomedical community

- peer reviewed and published immediately upon acceptance

- cited in PubMed and archived on PubMed Central

- yours - you keep the copyright

Submit your manuscript here:

http://www.biomedcentral.com/info/publishing_adv.asp 